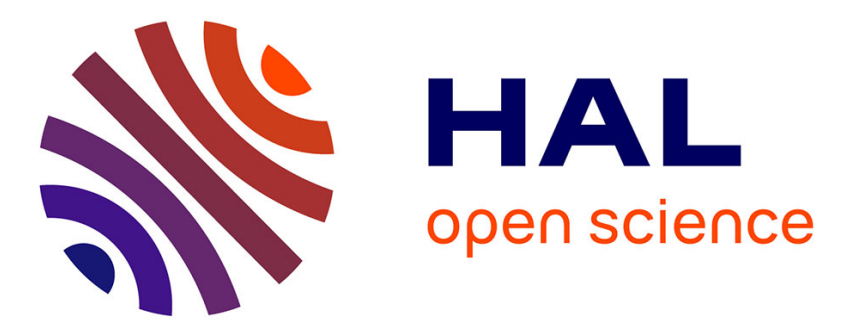

\title{
A $\mu$ TCA back-end firmware for data acquisition and slow control of the CLaRyS Compton camera
}

C. Caplan, O. Allegrini, J. P. Cachemiche, B. Carlus, X. Chen, D. Dauvergne, R. Della Negra, M. Fontana, L. Gallin-Martel, J. Hérault, et al.

\section{- To cite this version:}

C. Caplan, O. Allegrini, J. P. Cachemiche, B. Carlus, X. Chen, et al.. A $\mu$ TCA back-end firmware for data acquisition and slow control of the CLaRyS Compton camera. IEEE MIC 2019, Oct 2019, Manchester, United Kingdom. 10.1109/NSS/MIC42101.2019.9059941 • hal-02309672

\section{HAL Id: hal-02309672 \\ https://hal.science/hal-02309672}

Submitted on 20 Mar 2020

HAL is a multi-disciplinary open access archive for the deposit and dissemination of scientific research documents, whether they are published or not. The documents may come from teaching and research institutions in France or abroad, or from public or private research centers.
L'archive ouverte pluridisciplinaire HAL, est destinée au dépôt et à la diffusion de documents scientifiques de niveau recherche, publiés ou non, émanant des établissements d'enseignement et de recherche français ou étrangers, des laboratoires publics ou privés. 


\title{
A $\mu$ TCA back-end firmware for data acquisition and slow control of the CLaRyS Compton camera
}

\author{
C. Caplan, O. Allegrini, J.-P. Cachemiche, B. Carlus, X. Chen, D. Dauvergne, R. Della-Negra, M. Fontana, L. \\ Gallin-Martel, J. Hérault, D. Lambert, G.-N. Lu, M. Magne, H. Mathez, G. Montarou, C. Morel, \\ M. Rodo Bordera, E. Testa, Y. Zoccarato.
}

\begin{abstract}
With the aim to build a real-time controlled time-offlight Compton camera for an improved ion-range dose delivery control on hadrontherapy, as defined by the CLaRyS collaboration project, a programming data acquisition and slow control back-end electronics is necessary. This paper presents the FPGA based firmware architecture, embedded software and associated tools for the back-end electronics of CLaRyS, that is able to provide multiple $3 \mathrm{~Gb} / \mathrm{s}$ optical links for data acquisition, trigger distribution and slow control over its detectors. Performance of the system based on simulations and analysis of the results acquired during the characterization of a beam hodoscope will be presented. Experiments were performed with the $65 \mathrm{MeV}$ proton beam of the medical cyclotron at the Mediterranean Protontherapy Institute, in Nice.
\end{abstract}

Index Terms-DSP, FPGA, Compton camera, hadrontherapy.

\section{INTRODUCTION}

$\mathbf{P}$ ROTONTHERAPY and hadrontherapy using pencil beams of protons or carbon ions can deliver a dose more precisely as compared to traditional, X-ray based, radiotherapy. In practice, however, a major issue remains in the quality control of the treatment regarding the Bragg peak position and its alignment with the tumor volume.

Online monitoring of the Bragg peak position can be based on the detection of secondary radiation emitted after nuclear reactions of the incident particles within the tissues. It has been shown that the production rate of prompt-gamma ray emission, with an energy range of $1-10 \mathrm{MeV}$, is correlated to the range of the primary particles. For the purpose of range monitoring, different approaches for prompt gammaray imaging are currently being followed to monitor proton range. To explore these approaches, a mechanically collimated camera and a Compton camera allied with online control are proposed by the CLaRyS collaboration [1].

C. Caplan, J.-P. Cachemiche, C. Morel and M. Rodo Bordera are with the Aix-Marseille Univ, CNRS/IN2P3, CPPM, Marseille, France.

O. Allegrini, X. Chen, B. Carlus, L. Caponetto, R. Della-Negra, M. Fontana, H. Mathez, E. Testa and Y. Zoccarato are with Univ Claude Bernard Lyon I, CNRS/IN2P3, IPNL, Villeurbanne, France

D. Dauvergne and L. Gallin-Martel are with Univ Grenoble-Alpes, IN2P3/CNRS, LPSC, Grenoble, France.

G.-N. Lu is with Institute of Nanotechnologies of Lyon (INL), University of Lyon 1, IN2P3/CNRS, INL, Villeurbanne, France.

D. Lambert, M. Magne and G. Montarou are with Univ Clermont Auvergne, CNRS/IN2P3, LPC, Aubière, France.

J. Hérault is with Centre Antoine Lacassagne, Cyclotron biomédical, 227 av. de la Lanterne, 06200 Nice, France.

The authors would like to thank the support from ITMO-Cancer through the CLaRyS-UFT project, Labex PRIMES and the Region of Provence-Alpes-Cte d'Azur under the funding program "Emplois Jeunes Doctorants".

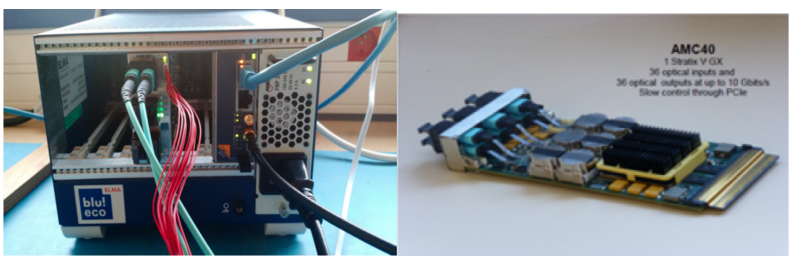

(a)

(b)

Fig. 1. 1a AMC40 DAQ board inside a $\mu \mathrm{TCA}$ crate, along with the $\mathrm{MCH}$ and the clock module and $1 \mathrm{~b}$ alone in the right.

The CLaRyS Compton camera is using three types of particle detectors: a BGO scintillation crystal absorber, a scintillating-fiber beam-tagging hodoscope and a silicon photon scatterer that must be all synchronized together by a control PC to ensure proper data acquisition, storage and analysis. To provide the necessary connectivity, physical data management, real-time slow control and low latency triggering between all these elements, programming data acquisition and slow control back-end electronics.

\section{PRoject SETUP AND ARCHITECTURE OF THE BACK-END FIRMWARE}

The specifications for the back-end electronics of the experiment were set to fulfill the necessary needs for the physical performing of the Compton camera at a reduced beamparticles rate compared to clinical levels of $10^{10}$ protons/s. The reason for this comes from the fact that too many events detected at the time by the camera would make it impossible to see the Bragg peak as it was shown with Geant4 simulations [2].

The electronic setup of the back-end electronics, illustrated in Figure 1a, is composed of a commercial $\mu$ TCA crate [3], a micro-TCA carrier hub $(\mathrm{MCH})$ with a Gigabit Ethernet switch and clock distribution support [4] and a custom inhouse developed advanced mezzanine card (AMC) with a large FPGA and multiple optical links which is called AMC40 [5], illustrated in 1b The AMC40 comprises essentially the following features:

- An Intel FPGA Stratix V SGXEA7N2F45C2 with $234 \mathrm{~K}$ logic cells;

- 36 optical fiber links and clock jitter cleaner;

- AMC backplane connector with support for a 1 Gigabit Ethernet link. 


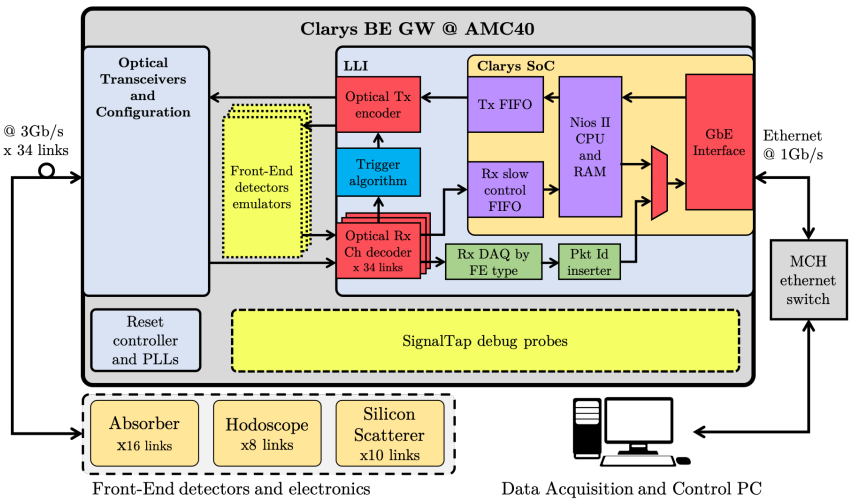

Fig. 2. Firmware architecture representation of the Back-End electronics of CLaRyS.

Following this setup, the bulk of this work is the implementation of the FPGA firmware and analysis of the performance of the Compton camera configuration. The block diagram on Figure 2 shows how the entire setup of the experiment is disposed, with the back-end on the central and top part, the three detector front-ends in the bottom left and the slow control and data acquisition PC on the bottom right.

The detectors are connected with the back-end FPGA on the AMC40 by 34 bidirectional optical links at a maximum data rate of $3 \mathrm{~Gb} / \mathrm{s}$ per link, which represents a theoretical peak of $102 \mathrm{~Gb} / \mathrm{s}$ in each direction. The connection with the $\mathrm{PC}$ is made by the $1 \mathrm{~Gb} / \mathrm{s}$ Ethernet link. A $40 \mathrm{MHz}$ clock signal synchronous along all the electronics elements of the chain is sourced by one of the Front-End boards through an SMA cable. Briefly the firmware is composed of VHDL code, QSYS and IP cores for the FPGA gateware part and by $\mathrm{C} / \mathrm{C}++$ code for the embedded firmware, which is divided in three datapaths: DAQ, for physical data acquisition, Slow Control for experiment control and monitoring of the detectors and electronics, and Trigger, to provide fast distribution of pretrigger and trigger frames for the triggering procedure. There are 6 transceivers responsible for the 36 bidirectional optical links, where up to 34 are used on the project making room for a future $10 \mathrm{~Gb} / \mathrm{s}$ Ethernet connections to the DAQ PC as an alternative to use the copper cable through the $\mathrm{MCH}$.

\begin{tabular}{|c|c|c|}
\hline \multicolumn{3}{|c|}{ Compilation information } \\
\hline $\begin{array}{l}\text { Software version } \\
\text { Device }\end{array}$ & $\begin{array}{l}\text { Quartus Prime Standard Editic } \\
\text { Stratix V 5SGXEA7N2F45C2 }\end{array}$ & $18.0 \mathrm{~B} 614$ \\
\hline \multicolumn{3}{|c|}{ Resources utilization } \\
\hline Logic utilization & $114601 / 234720$ & $49 \%$ \\
\hline Total block memory bits & $18533876 / 52428800$ bits & $35 \%$ \\
\hline Total RAM blocks & $1166 / 2560$ & $46 \%$ \\
\hline Total DSP blocks & $15 / 256$ & $6 \%$ \\
\hline Total PLLs & $45 / 92$ & $49 \%$ \\
\hline
\end{tabular}

COMPILATION SUMMARY OF THE FPGA PROJECT.

As most of the front-end detectors were not ready or available for tests during the framework of this project, there was a necessity to develop custom made detector emulators, on hardware (HDL), that could be embedded directly on the chip. Detector emulators at frame format conformance and basic functional level were developed for the Hodoscope and Absorbers types, represented on Figure 2 by the blocks "FrontEnd detectors emulators".

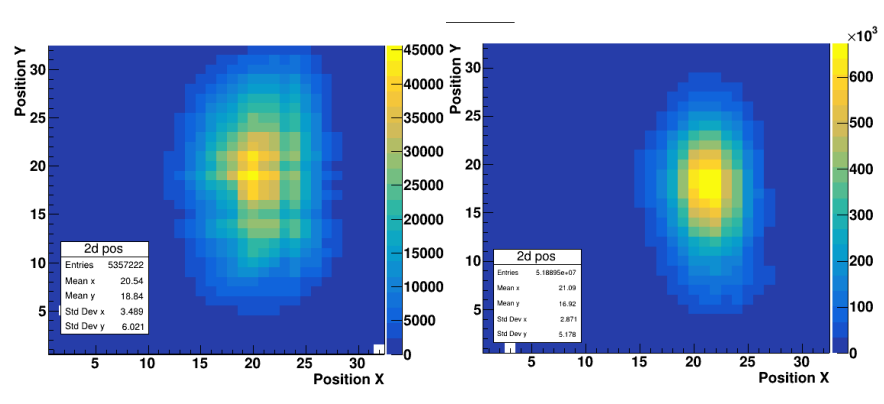

(a)

(b)

Fig. 3. 2D beam profiles with a coincidence rate of $1.3 \mathrm{MHz}$ in Figure $3 \mathrm{a}$ and at the rate of $20 \mathrm{MHz}$ in Figure $3 \mathrm{~b}$

\section{RESULTS AND ANALYSIS}

The CLaRyS prompt gamma imaging project was tested with various partial setup assemblies during at two beam tests in Sep 2018 and Mar 2019, with the $65 \mathrm{MeV}$ proton beam of the MEDICYC cyclotron of the Mediterranean Protontherapy Institute in Nice, France. Characterization of the detector's performance is currently underway. Figures 3 presents a twodimensional beam profile acquired in March 2019 for two beam intensities, achieving a triggering rate of $1.3 \mathrm{MHz}$.

On the measured features, the round-trip time (RTT) for Trigger operations is 26 clock cycles of $150 \mathrm{MHz}$, approximately $173.5 \mathrm{~ns}$. Slow control operations are verified to be in the 1-100 ms range. Data on resource utilization of the FPGA are presented in Table I.

\section{CONCLUSION}

The back-end DAQ and slow control electronics of the CLaRyS project proved to be mature, functional and conformant to the needs of trigger, physical data acquisition and slow control of the detectors during two beam tests with $65 \mathrm{MeV}$ protons from the MEDICYC cyclotron of the Mediterranean Protontherapy Institute in Nice. Based on embedded emulator simulations and experimental measurements, the performance of the back-end DAQ and slow control is currently being thoroughly analyzed and characterized and will be presented shortly at the conference.

\section{REFERENCES}

[1] J. Krimmer et al., "Development of a compton camera for medical applications based on silicon strip and scintillation detectors," Nucl. Instr. Meth. Phys. Res. A, vol. 787, pp. 98-101, 2015.

[2] M. Fontana et al., "Compton camera study for high efficiency spect and benchmark with anger system," Phys. Med. Biol., vol. 62, no. 23, 2017.

[3] "MicroTCA, COMBlue," https://www.elma.com/fr-eu/products/systemssolutions/chassis-platforms/product-pages/microtca/microtca-blueco/?sc_ lang=en accessed: 2010-05-01.

[4] "NAT-MCH - Mezzanine Modules," https://www.nateurope.com/ products/NAT-MCH\%20-\%20Mezzanine\%20Modules.html accessed: 2010-05-01.

[5] J.-P. Cachemiche et al., "Study for the $\mathrm{LHCb}$ upgrade read-out board," J. Instrum., vol. 5, no. 12, pp. C12036-C12036, dec 2010. [Online]. Available: https://doi.org/10.1088\%2F1748-0221\%2F5\% $2 \mathrm{~F} 12 \% 2 \mathrm{Fc} 12036$ 